\title{
Study of Higgs couplings to leptons and Higgs CP properties at the ILC
}

\author{
Daniel Jeans $^{* \dagger}$ \\ IPNS, KEK \\ E-mail: daniel.jeans@kek.jp
}

\begin{abstract}
The International Linear Collider (ILC) is a proposed electron positron collider providing 250 $\mathrm{GeV}$ collisions at with a luminosity of $\sim 10^{34} \mathrm{~cm}^{-2} \mathrm{~s}^{-1}$, producing $0.5 \times 10^{6}$ Higgs bosons. In this contribution we describe how the ILC can measure the Higgs boson coupling to leptons, and its CP properties via its decay to $\tau$ leptons. That the product of the Higgs production cross-section and its branching ratio $(\sigma \times B R)$ can be measured to a precision of around $1.2 \%$ for $h \rightarrow \tau^{+} \tau^{-}$, $20 \%$ for $h \rightarrow \mu^{+} \mu^{-}$, and that the mixing angle between odd and even components of the Higgs boson can be determined to $75 \mathrm{mrad}$.
\end{abstract}

The 39th International Conference on High Energy Physics (ICHEP2018)

4-11 July, 2018

Seoul, Korea

\footnotetext{
* Speaker.

†Talk given on behalf of the International Large Detector concept group.
} 


\section{Introduction}

The International Linear Collider (ILC) [1] is a planned electron-positron collider, initially providing $250 \mathrm{GeV}$ collisions, with polarised beams. The linear arrangement of the ILC allows future energy upgrades by lengthening the accelerators and/or by future improvements in accelerating technology.

The main aims of the $250 \mathrm{GeV}$ ILC stage are investigations of the electro-weak sector via comprehensive and model independent measurements of the Higgs boson's properties and highprecision studies of other electro-weak processes. In this contribution, we describe prospects for measurements using Higgs decays to leptonic final states.

\section{International Large Detector}

The International Large Detector (ILD) is one of two detector concepts being developed for ILC [2]. It is a high-precision detector optimised for Particle Flow event reconstruction (PFA), built around a charged particle tracking system made up of a vertex detector, a large time projection chamber, and additional silicon layers, within a $3.5 \mathrm{~T}$ magnetic field. Calorimeters with highly segmented readout provide the pattern recognition capabilities needed for optimal application of PFA. The detector will provide impact parameter precision $\sigma_{\mathrm{d} 0} \sim 5 \mu \mathrm{m}$, transverse momentum resolution $\sigma_{\mathrm{pT}} / \mathrm{p}_{\mathrm{T}} \sim 2 \times 10^{-5} \mathrm{p}_{\mathrm{T}}$, and jet energy resolution of $(3-4) \%$ over a wide range of jet energies.

The detector is implemented in a Geant4-based simulation model, together with a set of digitisation processors. The simulated signals are treated by different reconstruction codes to identify charged particle tracks, calorimeter clusters, and combined these into Particle Flow Objects (PFOs), corresponding to individual final state particles. The analyses presented in this contribution all use event samples subjected to such full simulation and reconstruction of ILD.

\section{Higgs Yukawa - mass relation}

In the Standard Model, the strength of the Yukawa coupling between the Higgs and fermions is predicted to be proportional to fermion masses. Different BSM models can predict variations in this relation, typically at the level of several \% for models with a new physics scale around a TeV. Measurements of couplings at the \% level or better will allow these BSM models to be probed. In this contribution we present projections on the measurement precision of muon and tau leptons' Yukawa couplings at ILC.

The cross-section measurement for the process $\mathrm{e}^{+} \mathrm{e}^{-} \rightarrow \mathrm{Zh}\left(\mathrm{h} \rightarrow \tau^{+} \tau^{-}\right)$was simulated in ILD. In the analysis described in [3, 4], decays of the $\mathrm{Z}$ boson into electrons, muons, and hadrons are considered. Tau lepton candidates are identified by searching for isolated narrow jets containing one or three charged particles, with a total charge of \pm 1 , and invariant mass below $2 \mathrm{GeV} / \mathrm{c}^{2}$.

Selection cuts are applied to reduce the contribution of background processes. The collinear approximation is applied, assuming that the $\tau$ decay neutrinos are collinear with the visible tau decay jet; the resulting tau pair invariant mass distribution is shown in Fig.1 (left). As a final step, a boosted decision tree is trained to distinguish signal from remaining background. 
The estimated measurement precision on the cross-section $\sigma\left(\mathrm{e}^{+} \mathrm{e}^{-} \rightarrow \mathrm{h}+\mathrm{X} ; \mathrm{h} \rightarrow \tau^{+} \tau^{-}\right)$is $1.2 \%$ for $2 \mathrm{ab}^{-1}$ of data at $250 \mathrm{GeV}$. Adding $4 \mathrm{ab}^{-1}$ of data at $500 \mathrm{GeV}$ improves the precision to $1.0 \%$.
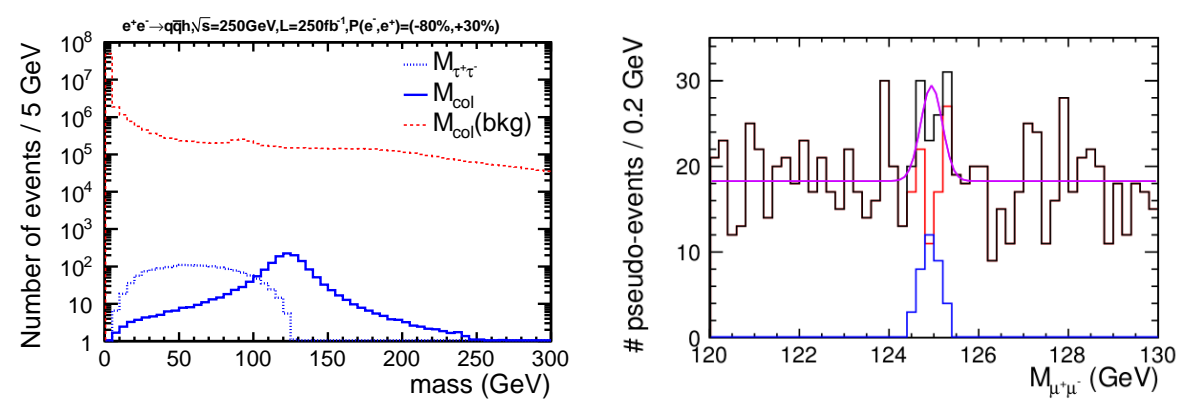

Figure 1: Left: Reconstructed tau pair invariant mass in $\mathrm{h} \rightarrow \tau^{+} \tau^{-}$analysis, when considering only visible decay products (signal only, dotted blue), the colinear approximation (signal only, solid blue), and when including all background processes (red) (from [3]). Right: A single pseudo-experiment in the $h \rightarrow \mu^{+} \mu^{-}$ analysis, showing the $\mu^{+} \mu^{-}$invariant mass. The signal is shown in blue and the background in red. Their sum is shown in black, and a fit to the distribution in pink.

The main challenge of the measurement of the muon Yukawa coupling is its small expected branching ratio, around $2 \times 10^{-4}$. A full simulation analysis [5] considers $h \rightarrow \mu^{+} \mu^{-}$production in association with a pair of neutrinos or quarks. Events are selected by requiring a pair of prompt, isolated, oppositely-charged and well-measured muon candidates. After a preselection considering the angles of the muon candidates and the activity in the remainder of the event, a multivariate discriminator is trained to distinguish signal from backgrounds.

The resulting di-muon invariant mass distribution is used to estimate the remaining number of signal events. A key aspect is the muon momentum measurement: the better it is, the sharper is the signal peak on the flat background distribution. The simulated distributions of selected signal and backgrounds are used to run a series of pseudo-experiments to estimate the expected statistical sensitivity. An example pseudo-experiment is shown in Fig.1 (right). Using $2 \mathrm{ab}^{-1}$ of data at $250 \mathrm{GeV}, \sigma\left(\mathrm{e}^{+} \mathrm{e}^{-} \rightarrow \mathrm{h}+\mathrm{X}, \mathrm{h} \rightarrow \mu^{+} \mu^{-}\right)$can be measured with a precision of $20 \%$, which improves to $15 \%$ when an additional $4 \mathrm{ab}^{-1}$ of data at $500 \mathrm{GeV}$ is included.

\section{CP measurement in $h \rightarrow \tau^{+} \tau^{-}$}

The decay into tau leptons of the Higgs boson provides access the CP nature of the Higgs and its couplings. The $\mathrm{CP}$ state of the tau pair affects transverse spin correlations between them, shown in Fig.2 (left). The distribution of $\tau$ decay products is sensitive to the $\tau$ polarisation, allowing these correlations to be measured. The high precision detectors envisaged for ILC and its clean experimental environment enable such a measurement.

Higgs bosons produced via Higgs-strahlung, and in which the $\mathrm{Z}$ decays to electrons, muons, or hadrons, were considered as signal. The impact parameters of tau decay products are used to help reconstruct the tau momenta, following the approach in [6]. Estimators of the tau spin direction, known as polarimeters, are simple to extract in the decays $\tau^{ \pm} \rightarrow \pi^{ \pm} v$ and $\tau^{ \pm} \rightarrow \pi^{ \pm} \pi^{0} v$. In [7], these two decay modes, accounting for $37 \%$ of tau decays, were used to analyse Higgs decays to 
tau leptons. After an event selection, events were classified according to their sensitivity to $\mathrm{CP}$ effects. Pseudo-experiments were run to estimate the experimental sensitivity, assuming $2 \mathrm{ab}^{-1}$ of data at $250 \mathrm{GeV}$ : the mixing angle between $\mathrm{CP}$ even and odd components of the tau-pair can be measured to a precision of $75 \mathrm{mrad}$.
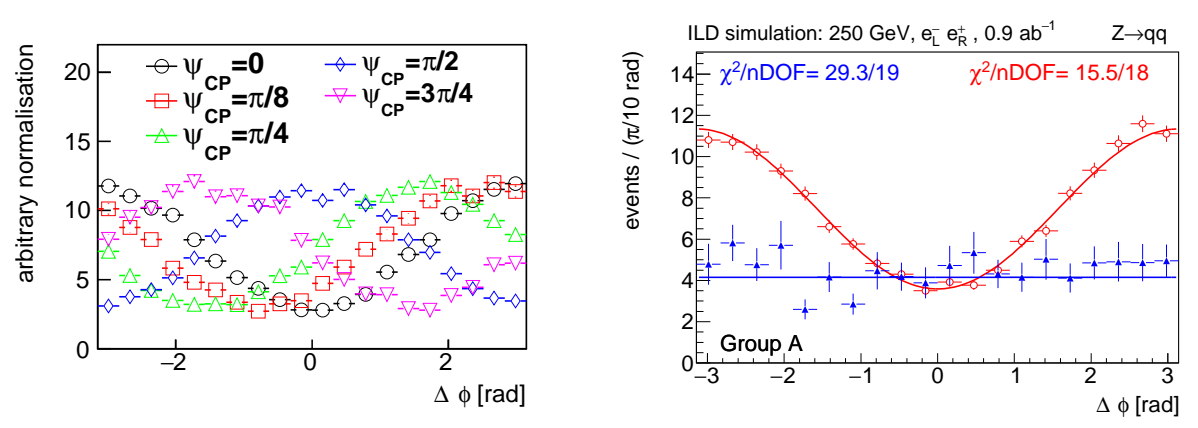

Figure 2: CP phase measurement in $h \rightarrow \tau^{+} \tau^{-}$. Left: distribution of the CP-sensitive correlation angle for different CP mixing cases, at MC level. Right: reconstructed angular distribution in the case of the SM, for a sub-sample of selected events; signal is shown in red, background in blue. Figures from [7].

\section{Acknowledgments}

We thank the LCC generator working group and ILD software working group for providing the simulation and reconstruction tools and Monte Carlo samples used in this study. This work has benefited from computing services provided by the ILC Virtual Organization, supported by the national resource providers of the EGI Federation and the Open Science GRID.

\section{References}

[1] C. Adolphsen et al., "The International Linear Collider Technical Design Report - Volume 3.II: Accelerator Baseline Design,” arXiv:1306.6328 [physics.acc-ph].

[2] T. Behnke et al., "The International Linear Collider Technical Design Report - Volume 4: Detectors," arXiv:1306.6329 [physics.ins-det].

[3] S. Kawada, K. Fujii, T. Suehara, T. Takahashi and T. Tanabe, "A study of the measurement precision of the Higgs boson decaying into tau pairs at the ILC", Eur. Phys. J. C 75, no. 12, 617 (2015).

[4] S. Kawada, "Study of Higgs boson decays to tau pairs at the International Linear Collider", Ph.D. thesis, Hiroshima University (2016). http://ir.lib.hiroshima-u.ac.jp/en/00040208.

[5] S. Kawada, J. List and M. Berggren, "Branching ratio measurement of $h \rightarrow \mu^{+} \mu^{-}$at the ILC," arXiv:1801.07966 [hep-ex].

[6] D. Jeans, "Tau lepton reconstruction at collider experiments using impact parameters," Nucl. Instrum. Meth. A 810, 51 (2016) [arXiv:1507.01700 [hep-ex]].

[7] D. Jeans and G. W. Wilson, "Measuring the CP state of tau lepton pairs from Higgs decay at the ILC," Phys. Rev. D 98, no. 1, 013007 (2018) [arXiv:1804.01241 [hep-ex]]. 